I.N.R.A.

BIBLIOTHEQUE UO 35906

DOMAINE DE CROUELLE

63039

CLERMONT-FD CEDEX?
I.N.R.A. AGRONOMIE

BIBIJOTHEQUE

Do:atume de Mo: Desir

63039 CLKKMONI FERRAND CEDEX

\title{
ERRATUM
} $7 / 2 / C O$

\section{Valeur alimentaire des marcs de raisin \\ I - Influence des 8 sources azotées de complémentation sur l'utilisation digestive par le mouton de marc de raisin épuisé et ensilé}

\author{
A. LARWENCE et A. YAHIAOUI \\ avec la collaboration technique de $\mathbf{K}$. DJoudad \\ Institut national agronomique, Département de Zootechnie \\ El Harrach, Alger
}

Page 363 lire :

Les matières azotées (sur produit frais), et les matières grasses sont déterminées selon les méthodes préconisées par le recueil des méthodes d'analyse des communautés européennes (1976), la cellulose brute selon la méthode de Weende, la qualité de l'ensilage est évaluée par la détermination de l'azote soluble après épuisement en triple d'un échantillon de $10 \mathrm{~g}$ ('azote soluble est dosé selon la méthode de Kjeldhal sur une partie aliquote de $10 \mathrm{ml}$ d'extrait), l'azote ammoniacal selon Conway (1965). Ces deux résultats sont exprimés par rapport à l'azote total déterminé sur le produit frais. Les acides acétique, butyrique et lactique sont dosés selon LEPPER (1938).

La solubilité et la fermentescibilité in vitro de l'azote de chaque composant des rations (à l'exception de l'urée) sont déterminées d'après les méthodes préconisées par I.N.R.A (1978).

Les tanins sont évalués après extraction par du méthanol absolu et précipitation par de l'éther éthylique selon MARTin-TANGUY et al. (1976).

\section{F. Calcul}

La valeur énergétique est déterminée en UFL. Nous avons estimé K1 à 60 p. 100 et la digestibilité de l'énergie par l'expression $\mathrm{dE}=1,0087$. $\mathrm{dMO}-0,0377$ (I.N.R.A., 1978).

Les résultats ont fait l'objet de comparaison de variances (test de Snedecor) avant d'éventuelles comparaisons de moyennes (test de Student). 


\section{Résultats et discussion}

Les résultats d'analyse présentés dans le tableau 3 sont en accord avec ceux rapportés par de nombreux auteurs cités par Matray, Aurejac \& Balmelle (1977), Ce sousproduit est par ailleurs particulièrement riche en tanins condensés (3,6 p. 100 de la matière sèche); cette teneur est 15 à 18 fois plus élevée que celle relicontrée dans la luzerne, le sorgho grain, le caroube et les féveroles riches en cette substance (MirJaVILA, 1979).

\section{A. Bilan de silos}

Les paramètres de conservation de l'ensilage sont rassemblés dans le tableau 3. Après une conservation de trois mois, les pertes par pourriture et les quantités d'acide acétique et d'ammoniac dosées sont faibles, respectivement 2,74 p. 100 , $7,84 \mathrm{~g}(/ \mathrm{kg} \mathrm{MS})$ et $0,17 \mathrm{p} .100$. Cependant le taux d'acide butyrique et le $\mathrm{pH}$ sont relativement élevés (respectivement $4,77 \mathrm{~g} / \mathrm{kg}$ de $\mathrm{MS}$ et 4,90 ).

\section{B. Solubilité et fermentescibilité des composants des rations}

Les matières azotées des marcs de raisin sont pratiquement insolubles (environ 2 p. 100) et très peu fermentescibles (9 p. 100). Dans ces conditions, la consommation de $3 \mathrm{~kg}$ de marc à $43 \mathrm{p} .100$ de matière sèche, n'apporte que $16 \mathrm{~g}$ de matières azotées fermentescibles (tabl. 4) ce qui paraît nettement insuffisant pour assurer une croissance normale des microorganismes du rumen. En effet, selon JaRRIGE, JouRNeT \& VERITE (1978), leurs besoins seraient assurés avec un fourrage bien consommé dosant 12 p. 100 de matières azotées dont 45 p. 100 sont fermentescibles, soit environ $54 \mathrm{~g}$.

\section{au lleu de:}

Cette insolubilité de l'azote des marcs de raisin dans le rumen ne semble pas pour autant augmenter la quantité de protéines d'origine alimentaire digestibles dans l'intestin (puisque les sujets consommant cet aliment en plat unique ont perdu en moyenne $130 \mathrm{~g}$ /jour) probablement à cause de sa forte teneur en tanins condensés (une consommation de $3000 \mathrm{~g}$ de marc représente une ingestion de $47 \mathrm{~g}$ de tanins) connus comme inhibiteurs des activités microbiennes et enzymatiques (TAGARI et al., 1965 ; FeENY, 1969), Ces substances phénoliques sont également responsables de l'insolubilisation de l'azote des marcs de raisin; des phénomènes similaires sont observés par LARWENCE (1979) sur des graines de féverole.

Les taux d'azote soluble et d'azote fermentescible enregistrés pour les sources azotées de complémentation (tabl. 4) sont conformes à ceux rapportés par DeMarquiLLY et al. (1978) sauf pour ce qui concerne les drêches de brasserie, pour lesquels nous obtenons un taux d'azote soluble plus élevé, et la féverole. Pour cette dernière, les mêmes auteurs donnent des valeurs plus élevées pour la fermentescibilité $(70$ p. 100 contre 30 p. 100). Il faut cependant noter que la fermentescibilité de l'azote de la féverole graine est très variable. Elle dépend du type de féverole (printemps ou hiver), de la variété, de sa teneur en azote et en tanins. Il existe une corrélation élevée $(R=-0,80$, $P<0,01$ ) entre fermentescibilité et teneur en tanins (LARWENCE, 1979). La féverole que nous avons utilisée est particulièrement riche en cette substance $(0,8$ p. 100 de la MS).

La valeur de fermentescibilité obtenue pour les fientes est assez surprenante compte tenu de la haute solubilité de leur azote ; la présence d'antibiotiques ou d'autres substances 
médicamenteuses perturbant l'activité des microorganismes, pourrait expliquer ce résultat. La cinétique de production d"ammoniac révèle un maximum $(50,2 \mathrm{p}, 100)$ après 8 heures d'incubation alors qu'il se situe à 95,4 p. 100 après seulement 2 heures pour l'urée.

\section{Digestibilité des rations}

Les résultats consignés dans le tableau 5 confirment la faible đigestibilité (respectivement $30,8,27,5$ et 19,3 pour la matière organique; la cellulose brute et les matières azotées) des marcs de raisin distribués en plat unique. Ils sont en accord avec ceux rapportés par de nombreux auteurs, notamment REYNE \& OARAMBoIs (1977) sur moutons et WolTer et al. (1979) sur Poney.

A l'image des marcs de pomme (ChARLET-Lery, Leroy \& ZetTER, 1955) et du tourteau d'olive (THERtez \& Boute, 1970), l'azote est le composant des marcs de raisin le moins digestible.

La complémentation azotée et énergétique falt augmenter de façon importante lutilisition digestive des rations (de 31 i 46 en moyenne) pour la matiere organique.

Cette amélloratlon ast llảe 目 la nature de la source azotée de complémentation. Alnsi, la digestlbllité de la matlềe organique variatzelle de 41 p. 100 (urée) 51 p. 100 (tourteau de colza). Néanmoins, quelle que soit la ration, l'ingestibilité est élevée puisqu'elle atteint $114 \mathrm{~g}$ de $\mathrm{MS} / \mathrm{kg} \mathrm{P}^{0,75}$, compte tenu que les refus enregistrés sont négligeables (tabl. 2). Les plus faibles digestibilités sont obtenues avec les drêches de brasserie (dont l'azote est peu fermentescible), la féverole (qui apporte $\mathbf{2} \mathrm{g}$ supplémentaires de tanins) et l'urée (peut-être trop rapidement dégradée et n'apportant pas de protéines). Ces trois régimes présentent des différences significatives $(\mathbf{P}<0,05)$ avec le tourteau de colza, le tourteau de soja et la luzerne.

\section{Page 364 lire :}

\section{TABLEAU 5}

Digestibilité des marcs de raisin avec ou sans concentré.

Apparent digestibility of grape marc with or without concentrate.

\begin{tabular}{l|r|r|c}
\hline \hline & $\begin{array}{c}\text { Marc } \\
\text { Grape marc }\end{array}$ & $\begin{array}{c}\text { Féverole } \\
\text { Field bean }\end{array}$ & $\begin{array}{c}\text { Soya } \\
\text { Soyabean meal }\end{array}$ \\
\hline & & & \\
Matière organique - Organic matter ... & $28,2 \pm 2,04$ & $42,1 \pm 2,03$ & $46,4 \pm 1,41$ \\
Matière organique - Organic matter ... & $30,8 \pm 2,00$ & $42,2 \pm 1,94^{*}$ & $47,0 \pm 1,79 * *$ \\
Cellulose brute - Crude fibre ......... & $27,5 \pm 1,73$ & $33,8 \pm 6,98$ & $41,0 \pm 2,41$ \\
Matières azotées - Crude proteins ..... & $19,3 \pm 4,16$ & $43,3 \pm 3,77$ & $46,2 \pm 4,20$ \\
Matière grasse - Fat ............... & $48,9 \pm 1,21$ & $55,1 \pm 0,81$ & $60,7 \pm 7,56$ \\
\hline * NS. & \\
** P<0,05. Le lot urée est considéré comme lot témoin - The urea group was used as a control \\
\hline
\end{tabular}


Cette insolubilité de l'azote des marcs de raisin dans le rumen ne semble pas pour autant augmenter la quantité de protéines d'origine alimentaire digestibles dans l'intestin (puisque les sujets consommant cet aliment en plat unique ont perdu en moyenne $130 \mathrm{~g} /$ jour) probablement à cause de sa forte teneur en tanins condensés (une consommation de $3000 \mathrm{~g}$ de marc représente une ingestion de $47 \mathrm{~g}$ de tanins) connus comme inhibiteurs des activités microbiennes et enzymatiques (TAGARI et al., 1965 ; FeENY, 1969). Ces substances phénoliques sont également responsables de l'insolubilisation de l'azote des marcs de raisin; des phénomènes similaires sont observés par LARWENCE (1979) sur des graines de féverole.

Les taux d'azote soluble et d'azote fermentescible enregistrés pour les sources azotées de complémentation (tabl. 4) sont conformes à ceux rapportés par DEMarQuiLLY et al. (1978) sauf pour ce qui concerne les drèches de brasserie, pour lesquels nous obtenons un taux d'azote soluble plus élevé, et la féverole. Pour cette dernière, les mêmes auteurs donnent des valeurs plus élevées pour la fermentescibilité (70 p. 100 contre 30 p. 100). Il faut cependant noter que la fermentescibilité de l'azote de la féverole graine est très variable. Elle dépend du type de féverole (printemps ou hiver), de la variété, de sa teneur en azote et en tanins. Il existe une corrélation élevée $(R=-0,80$; $\mathbf{P}<0,01$ ) entre fermentescibilité et teneur en tanins (LARWENCE, 1979). La féverole que nous avons utilisée est particulièrement riche en cette substance $(0,8$ p. 100 de la MS).

La valeur de fermentescibilité obtenue pour les fientes est assez surprenante compte tenu de la haute solubilité de leur azote ; la présence d'antibiotiques ou d'autres substances médicamenteuses perturbant l'activité des microorganismes, pourrait expliquer ce résultat. La cinétique de production d'ammoniac révèle un maximum $(50,2$ p. 100) après 8 heures d'incubation alors qu'il se situe à 95,4 p. 100 après seulement 2 heures pour l'urée.

\section{Digestibilité des rations}

Les résultats consignés dans le tableau 5 confirment la faible digestibilité (respectivement $30,8,27,5$ et 19,3 pour la matière organique; la cellulose brute et les matières azotées) des marcs de raisin distribués en plat unique. Ils sont en accord avec ceux rapportés par de nombreux auteurs, notamment REYNE \& GARAMBoIs (1977) sur moutons et Wolter et al. (1979) sur Poney.

A l'image des marcs de pomme (Charlet-Lery, Leroy \& ZelTer, 1955) et du tourteau d'olive (Theriez \& Boule, 1970), l'azote est le composant des marcs de raisin le moins digestible.

La complémentation azotée et énergétique fait augmenter de façon importante l'utilisation digestive des rations (de 31 à 46 en moyenne) pour la matière organique.

Cette amélioration est liée à la nature de la source azotée de complémentation. Ainsi, la digestibilité de la matière organique varie-t-elle de 41 p. 100 (urée) à 51 p. 100 (tourteau de colza). Néanmoins, quelle que soit la ration, l'ingestibilité est élevée puisqu'elle atteint $114 \mathrm{~g}$ de $\mathrm{MS} / \mathrm{kg} \mathrm{P}^{0,75}$, compte tenu que les refus enregistrés sont négligeables (tabl. 2). Les plus faibles digestibilités sont obtenues avec les drêches de brasserie (dont l'azote est peu fermentescible), la féverole (qui apporte $2 \mathrm{~g}$ supplémentaires de tanins) et l'urée (peut-être trop rapidement dégradée et n'apportant pas de protéines). Ces trois régimes présentent des différences significatives $(\mathrm{P}<0,05)$ avec le tourteau de colza, le tourteau de soja et la luzerne. 


\section{au lieu de :}

\section{TABleaU 5}

Digestibilité des marcs de raisin avec ou sans concentré.

Apparent digestibility of grape marc with or without concentrate.

\begin{tabular}{|c|c|c|c|}
\hline & $\begin{array}{c}\text { Marc } \\
\text { Grape marc }\end{array}$ & $\begin{array}{l}\text { Féverole } \\
\text { Field bean }\end{array}$ & $\begin{array}{c}\text { Soya } \\
\text { Soyabean meal }\end{array}$ \\
\hline Matière organique - Organic matter ... & $28,2 \pm 2,04$ & $42,1 \pm 2,03$ & $46,4 \pm 1,41$ \\
\hline Matière organique - Organie matter ... & $30,8 \pm 2,00$ & $42,2 \pm 1,94^{*}$ & $47,0 \pm 1,79 * *$ \\
\hline Cellulose brute - Crude fibre ......... & $27,5 \pm 1,73$ & $33,8 \pm 6,98$ & $41,0 \pm 2,41$ \\
\hline Matières azotées - Crude proteins & $19,3 \pm 4,16$ & $43,3 \pm 3,77$ & $46,2 \pm 4,20$ \\
\hline Matière grasse $-F a t$ & $48,9 \pm 1,21$ & $55,1 \pm 0,81$ & $60,7 \pm 7,56$ \\
\hline
\end{tabular}

* NS.

*: $\mathrm{P}<0,05$. Le lot urée est considéré comme lot témoin - The urea group was used as a control.

Les matières azotées (sur produit frais), et les matières grasses sont déterminées selon les méthodes préconisées par le recueil des méthodes d'analyse des communautés européennes (1976), la cellulose brute selon la méthode de Weende, la qualité de l'ensilage est évaluée par la détermination de l'azote soluble après épuisement en triple d'un échantillon de $10 \mathrm{~g}$ (l'azote soluble est dosé selon la méthode de Kjeldhal sur une partie aliquote de $10 \mathrm{ml}$ d'extrait), l'azote ammoniacal selon Conway (1965). Ces deux résultats sont exprimés par rapport à l'azote total déterminé sur le produit frais. Les acides acétique, butyrique et lactique sont dosés selon LEPPER (1938).

La solubilité et la fermentescibilité in vitro de l'azote de chaque composant des rations (à l'exception de l'urée) sont déterminées d'après les méthodes préconisées par I.N.R.A (1978).

Les tanins sont évalués après extraction par du méthanol absolu et précipitation par de l'éther éthylique selon Martin-TanguY et al. (1976).

\section{F. Calcul}

La valeur énergétique est déterminée en UFL. Nous avons estimé $\mathrm{K} l$ à 60 p. 100 et la digestibilité de l'énergie par l'expression $\mathrm{dE}=1,0087$. $\mathrm{dMO}-0,0377$ (I.N.R.A., 1978).

Les résultats ont fait l'objet de comparaison de variances (test de Snedecor) avant d'éventuelles comparaisons de moyennes (test de Student). 


\section{Page 365 llire:}

(Sulte du tableau 5)

\begin{tabular}{c|c|c|c|c|c}
\hline $\begin{array}{c}\text { Colza } \\
\begin{array}{c}\text { Rapeseed } \\
\text { oll meal }\end{array}\end{array}$ & $\begin{array}{c}\text { Luzerne } \\
\text { Dehydrated } \\
\text { lucerne }\end{array}$ & $\begin{array}{c}\text { P.O.U. } \\
\text { Sigle cell } \\
\text { protein }\end{array}$ & $\begin{array}{c}\text { Drtches } \\
\text { Brewer's } \\
\text { dried grains }\end{array}$ & $\begin{array}{c}\text { Urée } \\
\text { Urea }\end{array}$ & $\begin{array}{c}\text { Fientes } \\
\text { poultry } \\
\text { dropplings }\end{array}$ \\
\hline $48,8 \pm 2,40$ & $46,5 \pm 7,00$ & $46,0 \pm 2,37$ & $40,6 \pm 1,06$ & $40,3 \pm 4,54$ & $50,1 \pm 5,97$ \\
$50,6 \pm 3,20^{* * 1 *}$ & $49,6 \pm 7,41^{* *}$ & $46,0 \pm 4,93^{*}$ & $42,2 \pm 1,32 *$ & $40,6 \pm 8,11$ & $45,5 \pm 2,39$ \\
$51,0 \pm 4,41$ & $48,7 \pm 6,96$ & $44,2 \pm 1,89$ & $39,0 \pm 1,29$ & $39,0 \pm 1,28$ & $41,2 \pm 0,96$ \\
$45,4 \pm 1,40$ & $41,2 \pm 3,51$ & $48,5 \pm 1,06$ & $37,9 \pm 8,88$ & $40,1 \pm 6,17$ & $38,0 \pm 0,22$ \\
$68,1 \pm 1,65$ & $65,2 \pm 1,75$ & $62,0 \pm 1,11$ & $60,3 \pm 0,92$ & $57,0 \pm 2,29$ & $56,2 \pm 5,23$ \\
\hline
\end{tabular}

Les meilleurs résultats sont done obrenus avec les règimes qul apportent au molns 45 de matì̀res azotées fermentescibles (mare plus coneentré) provenant de matlères azotếes protélques, sil l'on en juge d'après les valeurs trouvées pour le lot urée. Il y a en effet, en dehors du lot urée, une corrélation hautement significative $(R=0,82$, $\mathbf{P}<0,01)$ entre la digestibilité de la matière organique des rations et la quantité d'azote fermentescible apportée par le concentré de complémentation. Cette liaison n'est pas observée $(R=0,27)$ pour ce qui concerne la solubilité de l'azote.

L'équation qui lie la digestibilité de la matière organique (y) à la quantité de matière azotée fermentescible apportée par le concentré $(\mathrm{x})$ est la suivante :

$$
y=0,289 x+33,77
$$

Elle est illustrée par la figure 1.

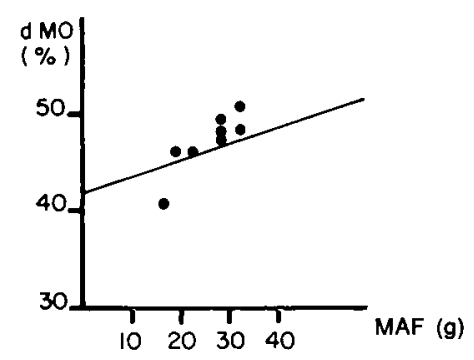

FIG. 1

Relation entre la digestibilité de la matière organique (dMO)

et la quantité de matières azotées fermentescibles apportées par chaque ration (MAF).

Relationship between organic matter digestibility (OMd)

and the amount of fermentescible crude protein supplied by each diet.

Par ailleurs, tous les principes nutritifs des rations voient leur digestibilité améliorée. L'utilisation digestive des matières azotées, de la cellulose brute, et des matières grasses, passe en moyenne respectivement de 19 à 42 p. 100 , de 27 à 42 p. 100 et de 49 à 
61 p. 100. Ce dernier élément est le plus digestible, sa digestibilité étant comparable à celle observée pour les fourrages de légumineuses ou de graminées. Toutefois, compte tenu de limportance des matières grasses dans les mares de raisin (environ 8 p. 100 de la MS), leur part dans la matière organique digestible représente 14 p. 100 alors qu'elle se situe entre 2 et 3 p. 100 pour les fourrages.

L'amélioration de la digestibilité des rations après complémentation ne résulte pas seulement de la digestibilité naturellement plus élevée du concentré et il est manifeste que la digestibilité propre des marcs de raisin s'est améliorée. En effet, en attribuant à la matière organique du concentré une digestibilité moyenne de $80 \mathrm{p}$. 100, le calcul montre que celle des marcs de raisin est passée de 31 p. 100 à 39 p. 100 (soit une augmentation de 26 p. 100).

Cette dernière valeur, 39 p. 100 , est comparable à celle observée pour la paille de blé ou d'orge distribuée sans complément. Avec $300 \mathrm{~g}$ de concentré, la digestibilité de la matière organique des rations à base de paille s'élève à 47 p. 100 (Dulphy, 1978); elle est inférieure de 9 p. 100 aux résultats que nous avons obtenus avec les marcs de raisin complémentés avec de la luzerne déshydratée ou du tourteau de colza.

au lleu de:

Apparent digestibility of grape marc with or withoul concentrate.

\begin{tabular}{|c|c|c|c|c|c|}
\hline $\begin{array}{c}\text { Colza } \\
\text { Rapeseed } \\
\text { oil meal }\end{array}$ & $\begin{array}{l}\text { Luzerne } \\
\text { Dehydrated } \\
\text { luccrne }\end{array}$ & $\begin{array}{l}\text { P.O.U. } \\
\text { Sigle cell } \\
\text { protein }\end{array}$ & $\begin{array}{c}\text { Dreches } \\
\text { Brewer's } \\
\text { dried grains }\end{array}$ & $\begin{array}{l}\text { Urée } \\
\text { Urea }\end{array}$ & $\begin{array}{l}\text { Fientes } \\
\text { poultry } \\
\text { droppings }\end{array}$ \\
\hline $48,8 \pm 2,40$ & $46,5 \pm 7,00$ & $46,0 \pm 2,37$ & $40,6 \pm 1,06$ & $40,3 \pm 4,54$ & $50,1 \pm 5,97$ \\
\hline $50,6 \pm 3,20^{\text {\#1* }}$ & $49,6 \pm 7,41^{\mathrm{km}}$ & $46,0 \pm 4,93^{*}$ & $42,2 \pm 1,32^{*}$ & $40,6 \pm 8,11$ & $45,5 \pm 2,39$ \\
\hline $51,0 \pm 4,41$ & $48,7 \pm 6.96$ & $44,2 \pm 1,89$ & $39,0=1,29$ & $39,0 \pm 1,28$ & $41,2 \pm 0,96$ \\
\hline $45,4 \pm 1,40$ & $41,2 \pm 3,51$ & $48,5 \pm 1,06$ & $37,9=8,88$ & $40,1 \pm 6,17$ & $38,0 \pm 0,22$ \\
\hline $68,1 \pm 1,65$ & $65,2 \pm 1,75$ & $62,0 \pm 1,11$ & $60,3 \pm 0,92$ & $57,0 \pm 2,29$ & $56,2 \pm 5,23$ \\
\hline
\end{tabular}

\section{Résultats et discussion}

Les résultats d'analyse présentés dans le tableau 3 sont en accord avec ceux rapportés par de nombreux auteurs cités par Matray, Aurejac \& Balmelle (1977). Ce sousproduit est par ailleurs particulièrement riche en tanins condensés (3,6 p. 100 de la matière sèche); cette teneur est 15 à 18 fois plus élevée que celle rencontrée dans la luzerne, le sorgho grain, le caroube et les féveroles riches en cette substance (MitJAVILA, 1979).

\section{A. Bilan de silos}

Les paramètres de conservation de l'ensilage sont rassemblés dans le tableau 3. 
Après une conservation de trois mois, les pertes par pourriture et les quantités d'acide acétique et d'ammoniac dosées sont faibles, respectivement 2,74 p. 100 , $7,84 \mathrm{~g}(/ \mathrm{kg} \mathrm{MS})$ et $0,17 \mathrm{p} .100$. Cependant le taux d'acide butyrique et le $\mathrm{pH}$. sont relativement élevés (respectivement $4,77 \mathrm{~g} / \mathrm{kg}$ de $\mathrm{MS}$ et 4,90 ).

B. Solubilité et fermentescibilité des composants des rations

Les matières azotées des marcs de raisin sont pratiquement insolubles (environ 2 p. 100) et très peu fermentescibles $(9$ p. 100). Dans ces conditions, la consommation de $3 \mathrm{~kg}$ de marc à $43 \mathrm{p}$. 100 de matière sèche, n'apporte que $16 \mathrm{~g}$ de matières azotées fermentescibles (tabl. 4) ce qui paraît nettement insuffisant pour assurer une croissance normale des microorganismes du rumen. En effet, selon JARRIGE, JouRnet \& Verite (1978), leurs besoins seraient assurés avec un fourrage bien consommé dosant 12 p. 100 de matières azotées dont 45 p. 100 sont fermentescibles, soit environ $54 \mathrm{~g}$. 


\title{
Valeur alimentaire des marcs de raisin \\ I - Influence des 8 sources azotées de complémentation sur l'utilisation digestive par le mouton de marc de raisin épuisé et ensilé
}

\author{
A. LARWENCE et A. YAHIAOUI \\ avec la collaboration technique de K. DJoudad \\ Institut national agronomique, Département de Zootechnie \\ El Harrach, Alger
}

\begin{abstract}
Résumé
Nous avons étudié l'influence de 8 sources azotées de complémentation (urée, féverole, drèches de brasserie, tourteau de soja, fientes de volailles, protéines d'organismes unicellulaires, luzerne déshydratée et tourteau de colza) sur la digestibilité chez le mouton des marcs de raisin épuisés et ensilés. Distribués seuls, l'utilisation digestive de la matière organique des mares de raisin est faible ( 31 p. 100), complémentés, elle passe de 40 p. 100 (urée) à 50 p. 100 (luzerne et tourteau de colza).

L'ingestibilité est particulièrement élevée; elle se situe entre 105 et $118 \mathrm{~g}$ de $\mathrm{MS} / \mathrm{kg}$ $\mathrm{P}^{0,75}$ suivant les régimes étudiés. Par ailleurs, la digestibilité des rations est étroitement liée $(\mathbf{R}=0,82 ; \mathbf{P}<0,01)$ à la quantité d'azote fermentescible apportée par les sources de protéines de complémentation.

La valeur énergétique calculée des rations distribuées $(0,50 \mathrm{UFL} / \mathrm{tête} / \mathrm{jour})$ s'est améliorée par suite d'une meilleure digestibilité des marcs de raisin; elle atteint 0,80 UFL pour les régimes complémentés respectivement avec le tourteau de colza et avec la luzerne déshydratée. Dans cette expérience, ces deux concentrés se présentent comme étant les meilleures sources de protéines de complémentation; ils ont permis d'obtenir un croît de $140 \mathrm{~g}$ par jour (contre 13 à $110 \mathrm{~g}$ pour les autres concentrés).

La valeur énergétique par $\mathrm{kg}$ de matière sèche de ration se situe pour ces deux régimes à 0,50 UFL, soit pratiquement autant que pour un foin moyen et plus que pour les pailles de céréale complémentées avec $300 \mathrm{~g}$ de concentré.

Les marcs de raisin épuisés et ensilés convenablement complémentés constituent donc un aliment intéressant pour des animaux à l'entretien ou à production modérée.
\end{abstract}

\section{Introduction}

Sur les 7 millions de tonnes de marcs de raisin épuisés disponibles annuellement dans le monde, moins de 3 p. 100 sont utilisés dans l'alimentation du bétail. Pour ce qui concerne l'Algérie qui en produit environ 100000 tonnes, ils sont entièrement jetés ou brûlés. Pourtant, leur composition chimique paraît intéressante comparativement à celle des fourrages classiques (cellulose brute, 23 p. 100 de la matière sèche ; matières 
azotées, 14 p. 100 ; cendres, 7 p. 100) bien que leur teneur en matières grasses soit sensiblement plus élevée (8 p. 100) (LARWENCE \& BerTHE, 1981).

Malheureusement, ces principes nutritifs sont peu digestibles; la digestibilité de la matière organique rapportée par Piccioni (1965), ReYNe \& Garambois (1977), DUMONT \& Tisserand (1978) s'élève respectivement à 30 p. 100, 28 p. 100 et 45 p. 100 (marc non distillé et déshydraté). La digestibilité des matières azotées selon les mêmes auteurs varie entre 9 et 19 p. 100. Par conséquent, les marcs de raisin épuisés distribués comme seul composant de la ration ne peuvent assurer les besoins azotés d'entretien des animaux. Certains auteurs, notamment Matray, Aurejac \& Balmelle (1977), conseillent en matière de rationnement de considérer comme nulle la digestibilité des matières azotées apportées par les marcs de raisin. Par ailleurs, LARWENCE \& BERTHE (1981) montrent que la solubilité de l'azote de l'ensilage de marcs est pratiquement nulle ( 2 p. 100 de l'azote total) et qu'un apport d'azote fermentescible $(15 \mathrm{~g}$ d'urée/tête/jour fait passer la digestibilité de la matière organique de 29 à 43 p. 100.

Les différentes sources de matières azotées pouvant être utilisées pour complémenter les marcs de raisin étant plus ou moins solubles et fermentescibles (GoERING \& Waldo, 1974 ; SNIFFEn, 1974 ; Demarquilly et al. (1978), il est utile de connaître celles qui sont le mieux adaptées à cette complémentation. C'est ce qui nous a conduit à comparer 8 sources azotées : féverole graine, tourteau de soja, tourteau de colza, luzerne déshydratée, protéines d'organismes unicellulaires (POU), drèches de brasserie, urée et fientes de volaille déshydratées.

\section{Matériel et méthode}

\section{A. Les aliments}

Le marc de raisin provient de la Mitidja et plus particulièrement de la commune de Mouzaïa. Il a subi une extraction d'alcool par le procédé vapeur. Le produit est conservé par ensilage sans conservateur dans des fûts de 200 litres. Sa valeur énergétique calculée (I.N.R.A., 1978) s'élève à 0,11 UFL/kg de MS.

Les régimes sont isoazotés (environ $64 \mathrm{~g}$ de matières azotées totales apportés par le concentré de complémentation, nous n'avons pas tenu compte de l'azote apporté par les marcs) et isoénergétiques (environ 0,50 UFL) à l'exception de celui d'un lot témoin d'animaux qui reçoit $4000 \mathrm{~g}$ de mares sans complémentation.

\section{B. Les animaux}

Les mesures sont effectuées sur des lots de 4 moutons mâles aduites non castrés de race Ouled-Djellal pesant en moyenne $36 \mathrm{~kg}(34$ à $37 \mathrm{~kg})$ placés en cage à métabolisme et maintenu à un niveau nutritionnel proche de celui de l'entretien $(64 \mathrm{~g}$ de matières azotées et $0,50 \mathrm{UFL} /$ jour).

\section{Déroulement des essais}

Durant trois semaines, les animaux sont accoutumés aux différents régimes expérimentaux (tabl. 1) distribués deux fois par jour ( 8 heures et 17 heures); la période de mesure dure deux semaines. 


\section{TABleaU 1}

Composition des régimes utilisés $(g)$.

Composition of diets $(g)$.

\begin{tabular}{|c|c|c|c|c|c|c|c|c|c|}
\hline $\begin{array}{l}\text { Lots - Groups } \\
\text { Aliments - Ingredients }\end{array}$ & I & II & III & IV & $\mathrm{V}$ & VI & VII & VIII & IX \\
\hline Marc de raisin-Grape marc .... & 4000 & 3000 & 3000 & 3000 & 3000 & 3000 & 3000 & 3000 & 3000 \\
\hline Maïs - Maize ............ & - & 125 & 230 & 180 & 240 & 230 & 135 & 300 & 225 \\
\hline Féverole graine - Field bean .... & - & 220 & - & 一 & 一 & - & - & - & - \\
\hline Tourteau de soja - Soyabean meal & - & - & 90 & - & - & 一 & - & 一 & 一 \\
\hline $\begin{array}{c}\text { Tourteau de colza - Rapeseed oil } \\
\text { meal } \ldots \ldots \ldots \ldots \ldots \ldots \ldots \ldots\end{array}$ & - & - & - & 125 & 一 & 一 & - & 一 & 一 \\
\hline 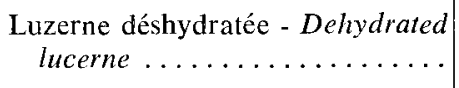 & - & 一 & - & - & 190 & 一 & 一 & 一 & - \\
\hline P.O.U. - Single cell protein . . . . & 一 & - & - & 一 & 一 & 75 & 一 & - & 一 \\
\hline $\begin{array}{c}\text { Drèches de brasseric - Brewer's } \\
\text { dried grains } \ldots \ldots \ldots \ldots \ldots \ldots\end{array}$ & 一 & 一 & - & - & - & - & 215 & 12 & 一 \\
\hline Urée - Urea & - & - & - & - & - & - & - & - & - \\
\hline $\begin{array}{r}\text { Fientes de volaille - Poultry drop- } \\
\text { pings } \ldots \ldots \ldots \ldots \ldots \ldots \ldots \ldots\end{array}$ & - & - & - & - & - & 一 & 一 & 一 & 2200 \\
\hline C.M.V. - Minerals and vitamins & 20 & 20 & 20 & 20 & 20 & 20 & 20 & 20 & 20 \\
\hline Soufre - Sulfur . & - & -- & - & - & - & 一 & 一 & - & - \\
\hline
\end{tabular}

D. Mesure

Les quantités de matière sèche ingérée sont mesurés chaque jour par pesée (à $1 \mathrm{~g}$ près) des quantités offertes et refusées (tab1. 2). Un échantillon représentatif de ce qui est distribué est constitué pour la période de mesure. Tous les matins, une récolte individuelle de fèces suivie d'une pesée a lieu ; un échantillon proportionnel est constitué dans un premier temps par jour et par animal et dans un deuxième temps par animal. et pour toute la période de mesure.

\section{E. Analyse}

La teneur en matière sèche (aliment et fèces) est déterminée par passage des échantillons à l'étuve ventilée à $80^{\circ} \mathrm{C}$, celle de l'ensilage de marc est corrigée (ScHOCH, 1949). pour tenir compte des pertes de produits volatils à l'étuve. 
TABleau 2

Matière sèche ingérée (g/j) moyenne cumulée de 25 jours.

Dry matter intake $(\mathrm{g} / \mathrm{d})$ mean of 25 days.

\begin{tabular}{|c|c|c|c|c|}
\hline Régimes - Diets & $\begin{array}{c}\text { Nombre } \\
\text { d'animaux } \\
\text { Number } \\
\text { of animals }\end{array}$ & $\begin{array}{c}\text { MS } \\
\text { distribuée } \\
D M \text { offered } \\
\end{array}$ & $\begin{array}{l}\text { MS refusée } \\
D M \text { refusal }\end{array}$ & $\begin{array}{l}\text { MS ingérée } \mathrm{g} / \mathrm{kg} \mathrm{P}^{0,75} \\
D M \text { intake } g / \mathrm{kg} W^{0.75}\end{array}$ \\
\hline $\begin{array}{l}\text { Marc de raisin } \ldots \ldots \ldots \\
\text { Grape marc }\end{array}$ & 4 & $\begin{array}{l}1724 \\
1724 \\
1724 \\
1724\end{array}$ & $\begin{array}{l}0 \\
0 \\
0 \\
0\end{array}$ & $\begin{array}{l}117 \\
115 \\
117 \\
119\end{array}$ \\
\hline Moyenne - Mean ..... & & 1724 & 0 & 117 \\
\hline Féverole - Field bean .... & 4 & $\begin{array}{l}1600 \\
1600 \\
1600 \\
1600\end{array}$ & $\begin{array}{r}17 \\
0 \\
21 \\
0\end{array}$ & $\begin{array}{l}112 \\
107 \\
107 \\
111\end{array}$ \\
\hline Moyerne - Mean ..... & & 1600 & 9 & 109 \\
\hline $\begin{array}{l}\text { Tourteau de soja } \\
\text { Soya bean meal }\end{array}$ & 4 & $\begin{array}{ll}1 & 574 \\
1 & 574 \\
1 & 574 \\
1 & 574 \\
\end{array}$ & $\begin{array}{c}0 \\
9 \\
23 \\
0\end{array}$ & $\begin{array}{l}109 \\
111 \\
103 \\
107\end{array}$ \\
\hline Moyenne - Mean ..... & & 1574 & 8 & 108 \\
\hline $\begin{array}{l}\text { Tourteau de colza } \ldots \ldots \\
\text { Rapeseed oil meal }\end{array}$ & 4 & $\begin{array}{l}1560 \\
1560 \\
1560 \\
1560\end{array}$ & $\begin{array}{r}0 \\
58 \\
41 \\
28\end{array}$ & $\begin{array}{l}108 \\
102 \\
108 \\
102\end{array}$ \\
\hline Moyerne - Mean ..... & & 1560 & 32 & 105 \\
\hline $\begin{array}{l}\text { Luzerne déshydratée } . . . \\
\text { Dehydrated lucerne }\end{array}$ & 4 & $\begin{array}{l}1671 \\
1671 \\
1671 \\
1671 \\
\end{array}$ & $\begin{array}{r}9 \\
38 \\
0 \\
19 \\
\end{array}$ & $\begin{array}{l}115 \\
116 \\
114 \\
110\end{array}$ \\
\hline Moyerne - Mean ..... & & 1671 & 16 & 114 \\
\hline P.O.U. - Single cell protein & 4 & $\begin{array}{l}1562 \\
1562 \\
1562 \\
1562 \\
\end{array}$ & $\begin{array}{r}8 \\
0 \\
0 \\
77 \\
\end{array}$ & $\begin{array}{l}110 \\
104 \\
106 \\
103\end{array}$ \\
\hline Moyenne - Mean ..... & & 1562 & 21 & 106 \\
\hline $\begin{array}{l}\text { Drèches de brasserie } . . . \\
\text { Brewer's dried grains }\end{array}$ & 4 & $\begin{array}{c}1614 \\
1614 \\
1614 \\
-\end{array}$ & $\begin{array}{r}0 \\
0 \\
103 \\
- \\
\end{array}$ & $\begin{array}{l}108 \\
110 \\
105 \\
-\end{array}$ \\
\hline Moyenne - Mean ..... & & 1614 & 34 & 107 \\
\hline Urée - Urea ............ & 4 & $\begin{array}{l}1567 \\
1567 \\
1567 \\
1567 \\
\end{array}$ & $\begin{array}{r}32 \\
49 \\
0 \\
22 \\
\end{array}$ & $\begin{array}{l}104 \\
101 \\
109 \\
110\end{array}$ \\
\hline Moyenne - Mean ..... & & 1567 & 26 & 106 \\
\hline $\begin{array}{l}\text { Fientes de volaille } \ldots \ldots \\
\text { Poultry droppings }\end{array}$ & 4 & $\begin{array}{l}1666 \\
1666 \\
1666 \\
1666 \\
\end{array}$ & $\begin{array}{r}0 \\
0 \\
23 \\
17 \\
\end{array}$ & $\begin{array}{l}118 \\
113 \\
109 \\
115\end{array}$ \\
\hline Moyerne - Mean ..... & & 1666 & 10 & 114 \\
\hline
\end{tabular}




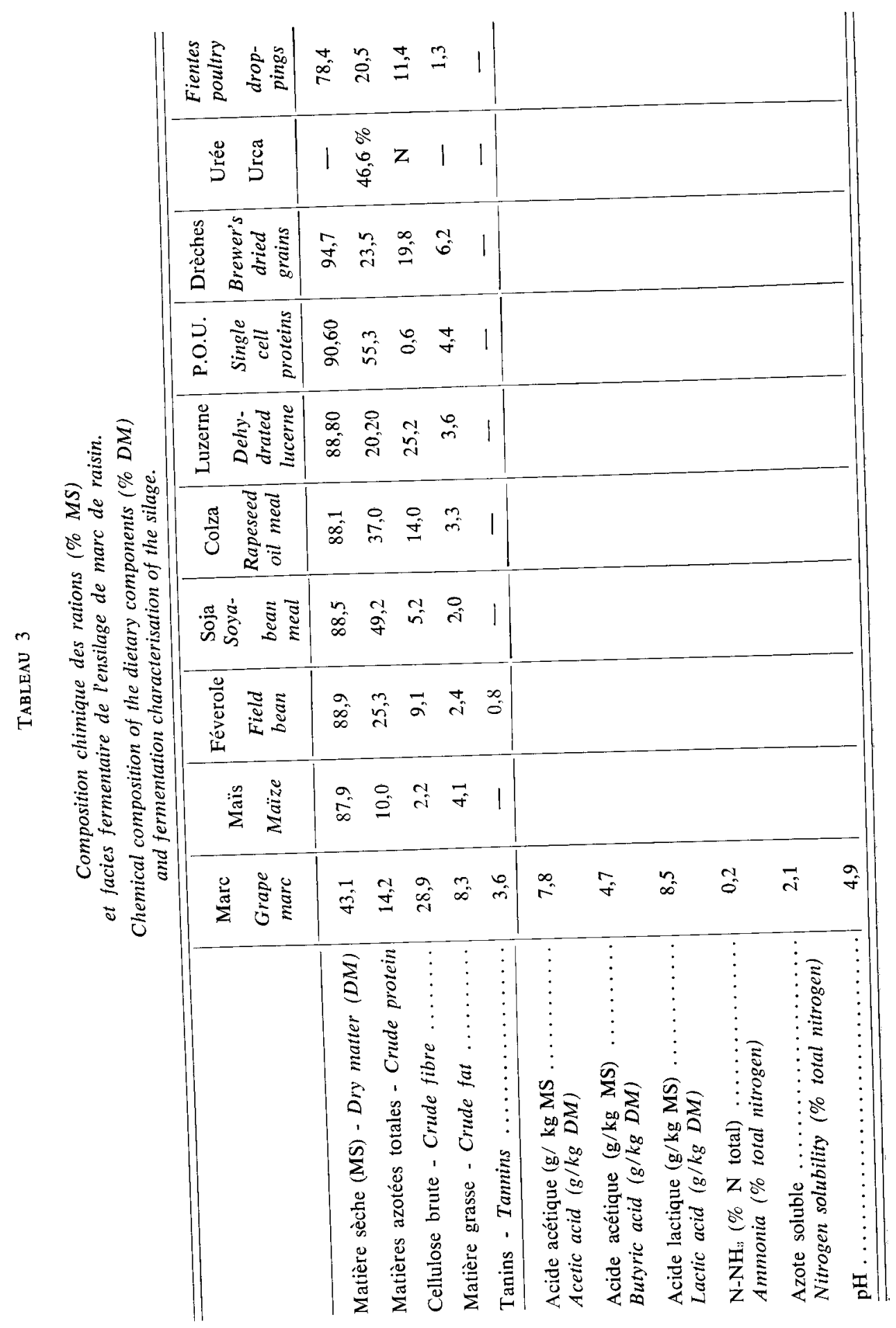




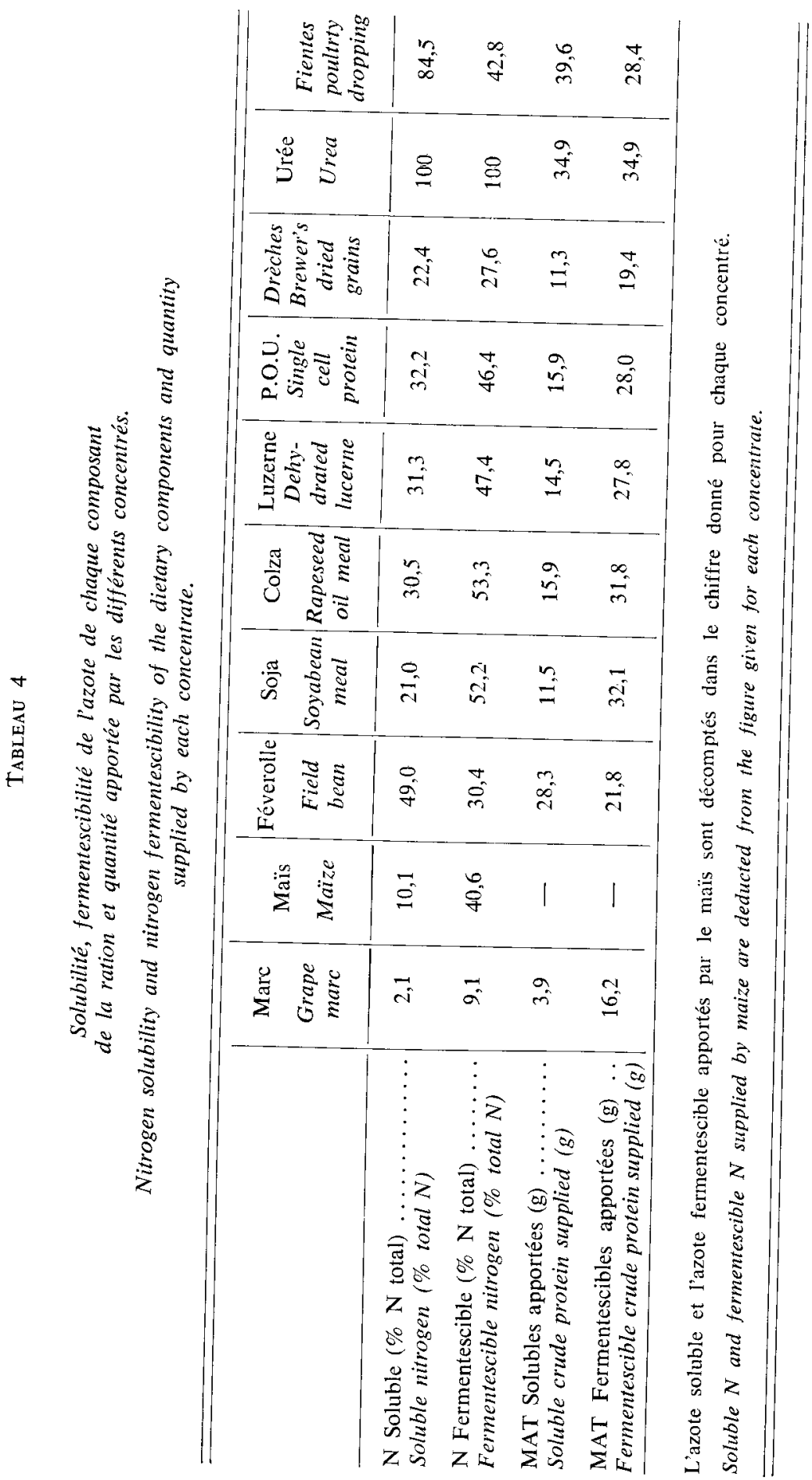


Cette insolubilité de l'azote des mares de raisin dans le rumen ne semble pas pour autant augmenter la quantité de protéines d'origine alimentaire digestibles dans l'intestin (puisque les sujets consommant cet aliment en plat unique ont perdu en moyenne $130 \mathrm{~g} /$ jour) probablement à cause de sa forte teneur en tanins condensés (une consommation de $3000 \mathrm{~g}$ de marc représente une ingestion de $47 \mathrm{~g}$ de tanins) connus comme inhibiteurs des activités microbiennes et enzymatiques (TAGARI et al., 1965 ; FeENY, 1969). Ces substances phénoliques sont également responsables de l'insolubilisation de l'azote des marcs de raisin; des phénomènes similaires sont observés par LARWENCE (1979) sur des graines de féverole.

Les taux d'azote soluble et d'azote fermentescible enregistrés pour les sources azotées de complémentation (tabl. 4) sont conformes à ceux rapportés par Demarquilly et al. (1978) sauf pour ce qui concerne les drèches de brasserie, pour lesquels nous obtenons un taux d'azote soluble plus élevé, et la féverole. Pour cette dernière, les mêmes auteurs donnent des valeurs plus élevées pour la fermentescibilité $(70$ p. 100 contre 30 p. 100). Il faut cependant noter que la fermentescibilité de l'azote de la féverole graine est très variable. Elle dépend du type de féverole (printemps ou hiver), de la variété, de sa teneur en azote et en tanins. Il existe une corrélation élevée $(R=-0,80$, $\mathbf{P}<0,01$ ) entre fermentescibilité et teneur en tanins (LARWEnce, 1979). La féverole que nous avons utilisée est particulièrement riche en cette substance $(0,8$ p. 100 de la MS).

La valeur de fermentescibilité obtenue pour les fientes est assez surprenante compte tenu de la haute solubilité de leur azote; la présence d'antibiotiques ou d'autres substances médicamenteuses perturbant l'activité des microorganismes, pourrait expliquer ce résultat. La cinétique de production d'ammoniac révèle un maximum $(50,2$ p. 100) après 8 heures d'incubation alors qu'il se situe à 95,4 p. 100 après seulement 2 heures pour l'urée.

\section{Digestibilité des rations}

Les résultats consignés dans le tableau 5 confirment la faible digestibilité (respectivement $30,8,27,5$ et 19,3 pour la matière organique ; la cellulose brute et les matières azotées) des marcs de raisin distribués en plat unique. Ils sont en accord avec ceux rapportés par de nombreux auteurs, notamment REYNE \& GARAMBoIs (1977) sur moutons et Wolter et al. (1979) sur Poney.

A l'image des mares de pomme (Charlet-Lery, Leroy \& Zelter, 1955) et du tourteau d'olive (Theriez \& Boule, 1970), l'azote est le composant des marcs de raisin le moins digestible.

La complémentation azotée et énergétique fait augmenter de façon importante l'utilisation digestive des rations (de 31 à 46 en moyenne) pour la matière organique.

Cette amélioration est liée à la nature de la source azotée de complémentation. Ainsi, la digestibilité de la matière organique varie-t-elle de 41 p. 100 (urée) à 51 p. 100 (tourteau de colza). Néanmoins, quelle que soit la ration, l'ingestibilité est élevée

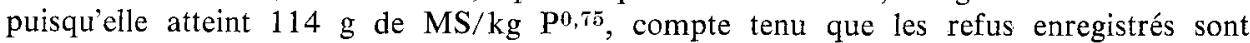
négligeables (tabl. 2). Les plus faibles digestibilités sont obtenues avec les drèches de brasserie (dont l'azote est peu fermentescible), la féverole (qui apporte $2 \mathrm{~g}$ supplémentaires de tanins) et l'urée (peut-être trop rapidement dégradée et n'apportant pas de protéines). Ces trois régimes présentent des différences significatives $(\mathbf{P}<0,05)$ avec le tourteau de colza, le tourteau de soja et la luzerne. 
Tableau 5

Digestibilité des marcs de raisin avec ou sans concentré.

Apparent digestibility of grape marc with or without concentrate.

\begin{tabular}{|c|c|c|c|}
\hline & $\begin{array}{c}\text { Mare } \\
\text { Grape marc }\end{array}$ & $\begin{array}{l}\text { Féverolc } \\
\text { Field bean }\end{array}$ & $\begin{array}{c}\text { Soya } \\
\text { Soyabean mea }\end{array}$ \\
\hline Matière organique - Organic matter ... & $28,2 \pm 2,04$ & $42,1 \pm 2,03$ & $46,4 \pm 1,41$ \\
\hline Matière organique - Organie matter ... & $30,8 \pm 2,00$ & $42,2 \pm 1,94^{*}$ & $47,0 \pm 1,79 *$ \\
\hline Cellulose brute - Crude fibre .......... & $27,5 \pm 1,73$ & $33,8 \pm 6,98$ & $41,0 \pm 2,41$ \\
\hline Matières azotées - Crude proteins ........ & $19,3 \pm 4,16$ & $43,3 \pm 3,77$ & $46,2 \pm 4,20$ \\
\hline Matière grasse $-F a t \ldots \ldots \ldots \ldots \ldots$ & $48,9 \pm 1,21$ & $55.1 \pm 0,81$ & $60,7 \pm 7,56$ \\
\hline \multicolumn{4}{|l|}{$*$ NS. } \\
\hline$* \quad \mathbf{P}<0,05$. Le lot urée est considéré comme lot & oin - The $u r$ & oup was used & a control. \\
\hline
\end{tabular}

Les matières azotées (sur produit frais), et les matières grasses sont déterminées selon les méthodes préconisées par le recueil des méthodes d'analyse des communautés européennes (1976), la cellulose brute selon la méthode de Weende, la qualité de l'ensilage est évaluée par la détermination de l'azote soluble après épuisement en triple d'un échantillon de $10 \mathrm{~g}$ (l'azote soluble est dosé selon la méthode de Kjeldhal sur une partie aliquote de $10 \mathrm{ml}$ d'extrait), l'azote ammoniacal selon Conway (1965). Ces deux résultats sont exprimés par rapport à l'azote total déterminé sur le produit frais. Les acides acétique, butyrique et lactique sont dosés selon LEPPER (1938).

La solubilité et la fermentescibilité in vitro de l'azote de chaque composant des rations (à l'exception de l'urée) sont déterminées d'après les méthodes préconisées par I.N.R.A (1978).

Les tanins sont évalués après extraction par du méthanol absolu et précipitation par de l'éther éthylique selon Martin-TanguY et al. (1976).

\section{F. Calcul}

La valeur énergétique est déterminée en UFL. Nous avons estimé K1 à 60 p. 100 et la digestibilité de l'énergie par l'expression $\mathrm{dE}=1,0087$. dMO - 0,0377 (I.N.R.A., 1978).

Les résultats ont fait l'objet de comparaison de variances (test de Snedecor) avant d'éventuelles comparaisons de moyennes (test de Student). 
(Suite du tableau 5)

\begin{tabular}{|c|c|c|c|c|c|}
\hline $\begin{array}{c}\text { Colza } \\
\text { Rapeseed } \\
\text { oil meal }\end{array}$ & $\begin{array}{c}\text { Luzerne } \\
\text { Dehydrated } \\
\text { lucerne }\end{array}$ & $\begin{array}{l}\text { P.O.U. } \\
\text { Sigle cell } \\
\text { protein }\end{array}$ & $\begin{array}{c}\text { Drèches } \\
\text { Brewer's } \\
\text { dried grains }\end{array}$ & $\begin{array}{l}\text { Urée } \\
\text { Urea }\end{array}$ & $\begin{array}{c}\text { Fientes } \\
\text { poultry } \\
\text { droppings }\end{array}$ \\
\hline $48,8 \pm 2,40$ & $46,5 \pm 7,00$ & $46,0 \pm 2,37$ & $40,6 \pm 1,06$ & $40,3 \pm 4,54$ & $50,1 \pm 5,97$ \\
\hline $50,6 \pm 3,20 * *$ & $49,6 \pm 7,41 * *$ & $46,0 \pm 4,93 *$ & $42,2 \pm 1,32^{*}$ & $40,6 \pm 8,11$ & $45,5 \pm 2,39$ \\
\hline $51,0 \pm 4,41$ & $48,7 \pm 6,96$ & $44,2 \pm 1,89$ & $39,0 \pm 1,29$ & $39,0 \pm 1,28$ & $41,2 \pm 0,96$ \\
\hline $45,4 \pm 1,40$ & $41,2 \pm 3,51$ & $48,5 \pm 1,06$ & $37,9 \pm 8,88$ & $40,1 \pm 6,17$ & $38,0 \pm 0,22$ \\
\hline $68,1 \pm 1,65$ & $65,2 \pm 1,75$ & $62,0 \pm 1,11$ & $60,3 \pm 0,92$ & $57,0 \pm 2,29$ & $56,2 \pm 5,23$ \\
\hline
\end{tabular}

\section{Résultats et discussion}

Les résultats d'analyse présentés dans le tableau 3 sont en accord avec ceux rapportés par de nombreux auteurs cités par Matray, Aurejac \& Balmelle (1977). Ce sousproduit est par ailleurs particulièrement riche en tanins condensés $(3,6$ p. 100 de la matière sèche); cette teneur est 15 à 18 fois plus élevée que celle rencontrée dans la luzerne, le sorgho grain, le caroube et les féveroles riches en cette substance (MITJAVILA, 1979).

\section{A. Bilan de silos}

Les paramètres de conservation de l'ensilage sont rassemblés dans le tableau 3 .

Après une conservation de trois mois, les pertes par pourriture et les quantités d'acide acétique et d'ammoniac dosées sont faibles, respectivement 2,74 p. 100, $7,84 \mathrm{~g}(/ \mathrm{kg}$ MS) et $0,17 \mathrm{p} .100$. Cependant le taux d'acide butyrique et le $\mathrm{pH}$ sont relativement élevés (respectivement $4,77 \mathrm{~g} / \mathrm{kg}$ de $\mathrm{MS}$ et 4,90 ).

\section{B. Solubilité et fermentescibilité des composants des rations}

Les matières azotées des marcs de raisin sont pratiquement insolubles (environ 2 p. 100) et très peu fermentescibles (9 p. 100). Dans ces conditions, la consommation de $3 \mathrm{~kg}$ de marc à 43 p. 100 de matière sèche, n'apporte que $16 \mathrm{~g}$ de matières azotées fermentescibles (tabl. 4) ce qui paraît nettement insuffisant pour assurer une croissance normale des microorganismes du rumen. En effet, selon JARRIGE, JourNet \& Verite (1978), leurs besoins seraient assurés avec un fourrage bien consommé dosant 12 p. 100 de matières azotées dont 45 p. 100 sont fermentescibles, soit environ $54 \mathrm{~g}$. 
Les meilleurs résultats sont donc obtenus avec les régimes qui apportent au moins $45 \mathrm{~g}$ de matières azotées fermentescibles (marc plus concentré) provenant de matières azotées protéiques, si l'on en juge d'après les valeurs trouvées pour le lot urée. Il y a en effet, en dehors du lot urée, une corrélation hautement significative $(R=0,82$, $\mathbf{P}<0,01)$ entre la digestibilité de la matière organique des rations et la quantité d'azote fermentescible apportée par le concentré de complémentation. Cette liaison n'est pas observée $(\mathrm{R}=0,27)$ pour ce qui concerne la solubilité de l'azote.

L'équation qui lie la digestibilité de la matière organique (y) à la quantité de matière azotée fermentescible apportée par le concentré $(\mathrm{x})$ est la suivante :

$$
y=0,289 x+33,77
$$

Elle est illustrée par la figure 1.

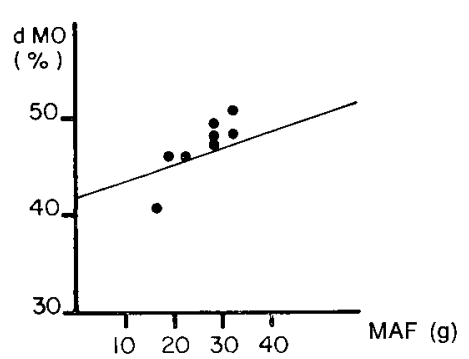

FIG. 1

Relation entre la digestibilité de la matière organique $(d M O)^{\circ}$

et la quantité de matières azotées fermentescibles apportées par chaque ration (MAF).

Relationship between organic matter digestibility (OMd)

and the amount of fermentescible crude protein supplied by each diet.

Par ailleurs, tous les principes nutritifs des rations voient leur digestibilité améliorée. L'utilisation digestive des matières azotées, de la cellulose brute, et des matières grasses, passe en moyenne respectivement de 19 à 42 p. 100 , de 27 à 42 p. 100 et de 49 à 61 p. 100 . Ce dernier élément est le plus digestible, sa digestibilité étant comparable à celle observée pour les fourrages de légumineuses ou de graminées. Toutefois, compte tenu de l'importance des matières grasses dans les marcs de raisin (environ 8 p. 100 de la MS), leur part dans la matière organique digestible représente 14 p. 100 alors qu'elle se situe entre 2 et 3 p. 100 pour les fourrages.

L'amélioration de la digestibilité des rations après complémentation ne résulte pas seulement de la digestibilité naturellement plus élevée du concentré et il est manifeste que la digestibilité propre des mares de raisin s'est améliorée. En effet, en attribuant à la matière organique du concentré une digestibilité moyenne de $80 \mathrm{p}$. 100, le calcul montre que celle des marcs de raisin est passée de 31 p. 100 à 39 p. 100 (soit une augmentation de 26 p. 100).

Cette dernière valeur, 39 p. 100 , est comparable à celle observée pour la paille de blé ou d'orge distribuée sans complément. Avec $300 \mathrm{~g}$ de concentré, la digestibilité de la matière organique des rations à base de paille s'éiève à 47 p. 100 (DuLPHY, 1978); elle est inférieure de 9 p. 100 aux résultats que nous avons obtenus avec les marcs de raisin complémentés avec de la luzerne déshydratée ou du tourteau de colza. 


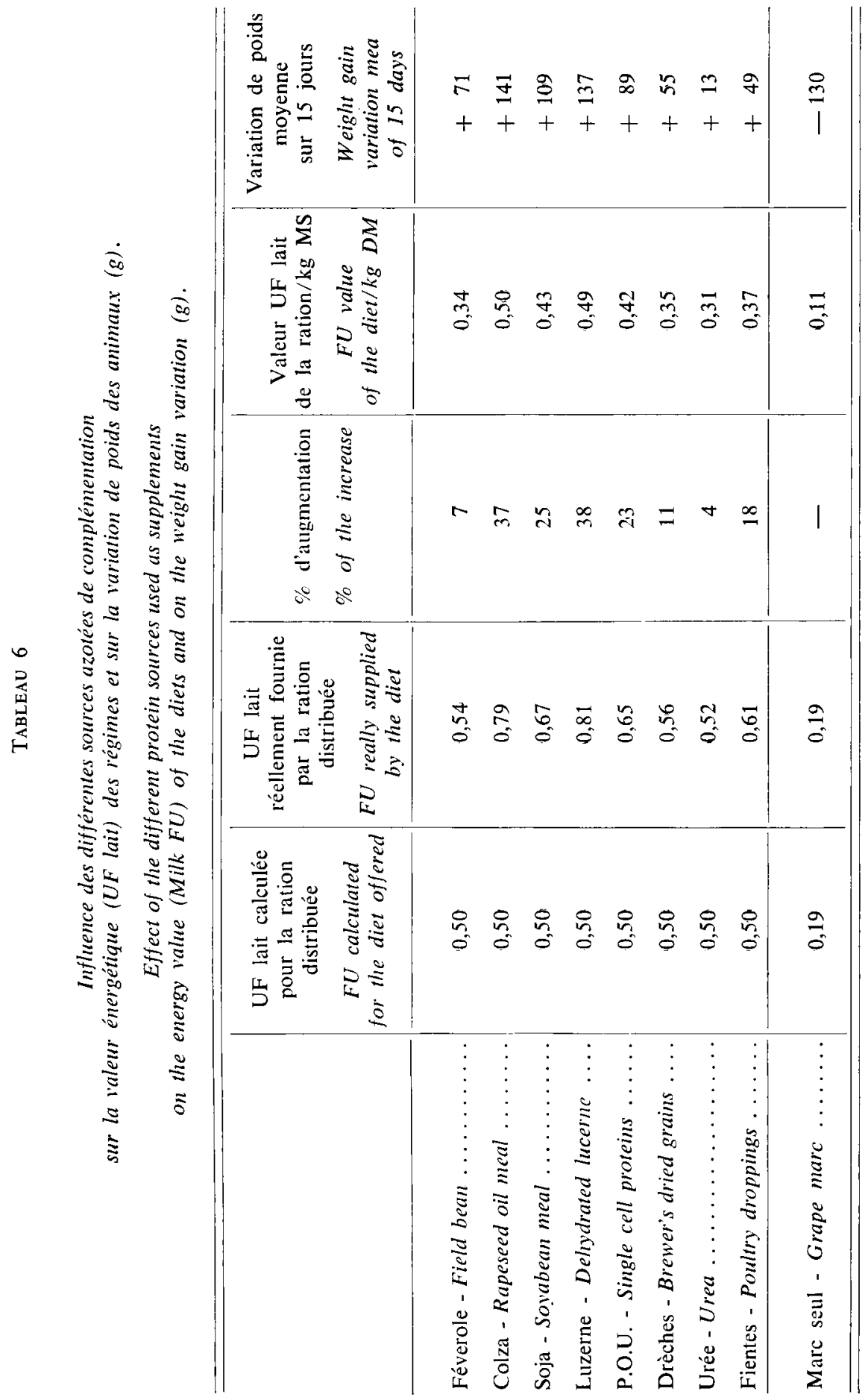




\section{Valeur énergétique des rations}

Le nombre d'UFL (calculé à partir de la digestibilité réelle des rations) réellement fourni par chaque ration est rapporté dans le tableau 6 : il varie de 0,47 UFL (urée) à 0,80 UFL (luzerne et colza). Sachant que les rations distribuées avaient une valeur énergétique théorique de 0,50 UFL, il s'est donc produit sous l'effet de la complémentation une amélioration appréciable de leur valeur énergétique (tableau 6), ce qui a permis aux animaux de prendre du poids durant la période expérimentale. Les gains de poids les plus élevés $(140 \mathrm{~g})$, sont obtenus avec les rations complémentées respectivement avec du tourteau de colza et de la luzerne déshydratée. Ce résultat est supérieur à celui obtenu (92 g) par CoRdesse \& TABA-TABAI (1981) avec des rations de paille traitée à l'ammoniac et complémentée avec $300 \mathrm{~g}$ de concentré.

Ces deux compléments sont en principe plus riches en soufre : le colza, car naturellement bien pourvu, la luzerne déshydratée, car enrichic en cet élément lors des opérations de déshydratation.

Le soufre pourrait jouer un rôle important dans les phénomènes de détoxification liés à la présence des substances phénoliques. Par ailleurs, selon Trenkle, Cheng \& Burroughs (1958); Evans \& Davis (1966), il stimule la digestion de la cellulose, résultats vérifiés pour ces deux régimes (tabl. 5). Pour les régimes uréc et fientes de volaille supplémentés en soufre, nous n’avons pas observé d'amélioration de la cellulolyse.

La valeur énergétique des rations par $\mathrm{kg}$ MS varie de 0,30 UFL (urée) à 0,50 UFL (luzerne et tourteau de colza). Elle dépasse 0,40 UFL pour le soja et les protéines d'organismes unicellulaires.

\section{Conclusion}

Les marcs de raisin distribués en plat unique sont très pauvres en énergie $(0,11$ UFL/kg de MS) et ne couvrent pas les besoins dentretien des animaux qui perdent du poids malgré une forte ingestion $\left(89 \mathrm{~g} / \mathrm{kg} \mathrm{P}^{\mathbf{n}, 75}\right)$. Un apport d'azote et d'énergie améliore de façon importante la valeur nutritive des rations; cette amélioration est cependant fonction de la nature de la complémentation qui doit fournir suffisamment d'azote fermentescible provenant d'une source de protéines vraies. Ainsi, la valeur énergétique des rations complémentées avec de l'urée et du maïs est-elle de $0,30 \mathrm{UFL} / \mathrm{kg}$ de MS alors qu'elle atteint $0,50 \mathrm{UFL}$ pour une complémentation par du tourteau de colza et de luzerne. Cette dernière valeur est comparable à celle observée pour des pailles de céréales complémentées avec $300 \mathrm{~g}$ de concentré.

Mais la quantité ingérée se situe à environ $900 \mathrm{~g}$ de MS pour la paille alors qu elle pourrait dépasser $1700 \mathrm{~g}$ pour les marcs de raisin puisque les refus enregistrés sont négligeables.

La valeur alimentaire de ce produit est donc supérieure à celle des pailles de céréale complémentées avec la même quantité de concentré. Il présente un intérêt certain pour des animaux à l'entretien ou à croissance modérée ; il présente en outre l'avantage, par rapport aux pailles, de ne pas nécessiter des frais de récolte, d'occuper peu de place et d'être facilement ensilable. 


\section{Summary \\ Feeding value of grape marc in ruminants}

\section{$I$ - Effect of the supplementing extracted grape marc silage with 8 protein sources on the digestibility in sheep}

The effect of supplementing extracted grape marc silage with 8 protein sources (urea, field bean, brewers dried grain, soyabean meal, poultry droppings, single cell proteins, dehydrated lucerne and rapeseed oil meal) on digestibility in sheep, was studied. Organic matter digestibility was low (31 p. 100) when grape marc was given alone, while it reached 40 p. 100 (urea) and 50 p. 100 (lucerne and rapeseed oil meal) when it was supplemented.

Voluntary feed intake was particularly high (between 105 and $118 \mathrm{~g} \mathrm{DM} / \mathrm{kg} \mathrm{W}^{0.75}$ ) according to the diet. Digestibility of the diets was closely related to the amount of fermentescible nitrogen supplied by the protein sources.

The energy value of the offered diets ( $0.50 \mathrm{Milk}$ FU/head/day) was improved because of the higher digestibility of grape marc. It reached 0.80 Milk FU for diets supplemented with rapeseed oil meal and dehydrated lucerne. In this experiment both concentrates appeared to be best protein sources. They led to a weight gain of $140 \mathrm{~g} / \mathrm{day}$ (versus 13 to $110 \mathrm{~g}$ for the other concentrates).

For these two diets the energy value per $\mathrm{kg} \mathrm{DM}$ was equal to $0.50 \mathrm{MFU}$, i.e. almost equivalent to that of a mean hay and higher than that of cereal straws supplemented with $300 \mathrm{~g}$ concentrate.

Accordingly properly supplemented extracted grape marc silage is a valuable feed for animals in maintenance or with a low production.

\section{Références bibliographiques}

Charlet-Lery G., Leroy A.M., Zelter S.Z., 1955. Etude chez le mouton et le porc de la digestibilité apparente des constituants de marcs de pomme frais, ensilés ou déshydratés. Ann. Zootech., 4, 321-332.

CONWAY E.J., 1965. Micro diffusion analysis and volumetric error. $5^{\circ}$ éd. Crosby Lockwood, London.

Cordesse R., Taba-Tabar M., 1981. Alimentation d'agneaux à partir de la paille traitée à l'ammoniac. I - Valeur nutritive, croissance et qualité des carcasses des animaux. Ann. Zootech., 30, 113-122.

Demarquilly C., Andrieu J., Sauvant D., Dulphy J.P., 1978. Composition et valeur nutritive des aliments. In': Alimentation des ruminants. Ed. I.N.R.A., route de SaintCyr, 78000 Versailles, $597 \mathrm{p}$.

DULPHY J.P., 1978. Valeur alimentaire des matières premières cellulosiques. In : Utilisation des matières premières cellulosiques, en particulier la paille, en alimentation animale. C.A.A.A., I.N.A., Paris-Grignon.

Dumont R., Tisserand J.L. ,1978. Valeur alimentaire d'un marc de raisin déshydraté. Ann. Zootech., 27, 631-637.

Evans J.L., Davis G.K., 1966. Dietary phosphorus, sulfur and molybdenum and mineral composition of rumen fluid. J. Anim. Sci., 25, 1010-1013.

FEENY P.P., 1969. Inhibitory effect of oak leaf tannins on the hydrolysis of proteins by trypsin. Phytochemistry, 8, 2119-2126.

Goering H.K., Weldo D.R., 1974. Processing effects on protein utilization by ruminants. Proc. Cornell nutrition conference, 25-36.

I.N.R.A., 1978. Alimentation des ruminants. Ed. I.N.R.A., route de Saint-Cyr, 78000 Versailles, $597 \mathrm{p}$. 
Jarrige R., Journet M., Verite R., 1978. Utilisation digestive et métabolique de l'azote. In : Alimentation des ruminants. Edl. I.N.R.A., route de Saint-Cyr, Versalles, pp. 89-128.

LARWence A., 1979. Mise au point d'un test permettant de classer des graines de féverole en fonction de leur teneur en tanins. Thèse de docteur-ingénieur, Université de Dijon.

Larwence A., Berthe J.L., 1981. Influence de deux sources de matières azotées (luzerne et uréc) sur la digestibilité par des moutons de marc de raisin épuisé et ensilé (ćtude du bilan azoté). Bull. soc. hist. nat. Afr. Nort, 69 (3-4), 61-73.

Lepper W., 1938. Technique de dosage des acides acétiques, butyrique et lactique dans les ensilages. Zeitschrift. Tierernïhr Futtermittek, (1), 187-196 (en allemand).

Martin-Tanguy J., Vermorel, M., Lenoble M., Martin C., 1976. Les tanins des graines de sorgho. Importance dans l'utilisation digestive de l'azote chez le rat en croissance. Ann. Biol. anim. Bioch. Biophys., 16, 879-890.

Matray M., Aurejac R., Balmelle J.C., 1977. Utilisation du marc de raisin épuisé dans l'alimentation hivernale des bovins. Publications I.T.E.B., Paris, $78 \mathrm{p}$.

Mitjavila S., 1979. Problèmes nutritionnels liés à la présence de tanins dans les aliments. Jourizées d'études du groupe polyphénols, 2-4 mai 1979.

Piccioni M., 1965. Dictionnaire des aliments pour les animaux. Edagricole, Bologne, $638 \mathrm{pp}$.

Recueil des méthodes d'analyse des communautés européennes, B.I.P.E.A. édit., 1976.

Reyne Y., Garambois X., 1977. Valeur alimentaire chez le mouton de l'ensilage de marc de raisin épuisé. Ann. Zootech., 26, 471-479.

Scносн W., 1949. Die bei der hocknung von sclageproben im trockenshranck auftrentenden verluste on flüchtigen Saüren und basen. Mitteilungen als' dem Gebrete der lebensmittelunlersuchung and Hygiène, 40, 170-189.

SNifFEN C.J., 1974. Nitrogen utilization as related to solubility of NPN and protein in feeds. Proc. Cornell Nutrition Conference, 12-18.

Tagari H., Henis Y., Tamir M., Volcani R., 1965. Effect of carob pod extract on cellulosis, proteolysis, deamination and protein biosynthesis in an artificiel rumen. Applied Microbiology, 13 (3), 437-442.

Theriez M., Boule G., 1970. Valeur alimentaire du tourteau d'olive. Ann. Zootech,, $19,143-157$.

Trenkle A.. Cheng E., Burroughs, 1958. Availability of different sulphur sources for rumen microorganisms in vitro cellulose digestion. J. Anim. Sci., 17, 1191-1198.

Wolter R., Durix A., Letourneau J.C., Calcelen M., 1979. Evaluation chez le poney de lat digestibilité du maïs, fourrage déshydraté, des pulpes sèches de betteraves, de la luzerne déshydratée, du son de blé, de là paille de blé et des pulpes de raisins. Ann. Zootech., 28, 93-100. 